FORMATION Formation emploi

Revue française de sciences sociales

122 | Avril-Juin 2013

Relever les défis de la Validation des Acquis de l'Expérience (VAE)

Présentation de l'ouvrage Former, certifier, insérer. Effets et paradoxes de l'injonction à la professionnalisation des diplômes

François Sarfati

\title{
OpenEdition
}

Journals

Édition électronique

URL : http://journals.openedition.org/formationemploi/4018

DOI : 10.4000/formationemploi.4018

ISSN : 2107-0946

Éditeur

La Documentation française

Édition imprimée

Date de publication : 30 juin 2013

Pagination : 167-169

ISSN : 0759-6340

Référence électronique

François Sarfati, «Présentation de l'ouvrage Former, certifier, insérer. Effets et paradoxes de l'injonction à la professionnalisation des diplômes », Formation emploi [En ligne], 122 | Avril-Juin 2013, mis en ligne le

10 juillet 2013, consulté le 30 octobre 2020. URL : http://journals.openedition.org/formationemploi/

4018 ; DOI : https://doi.org/10.4000/formationemploi.4018

(c) Tous droits réservés 


\title{
Note de lecture
}

\section{Présentation de l'ouvrage Former, certifier, insérer. Effets et paradoxes de l'injonction à la professionnalisation des diplômes}

\author{
par François Sarfati \\ chercheur au Centre d'études de l'emploi
}

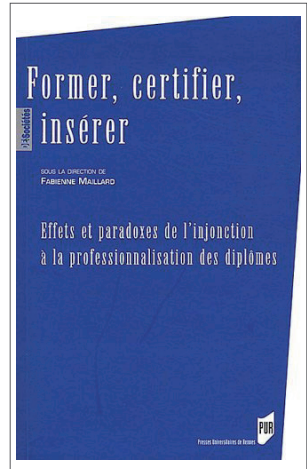

En 252 pages, cet ouvrage collectif renouvelle les travaux désormais classiques portés par Lucie Tanguy dans Lintrouvable relation formation-emploi (1986). Il s'agit pour les auteurs de prendre acte du retour de la pensée " adéquationniste" pour lancer une "réflexion sur ce qu'engage l'appel à la performance productive imposé aux diplômes, à ceux qui les élaborent, qui y forment, comme à ceux qui les détiennent" (p. 15). Le livre est pensé comme un espace de débat où des positions contrastées s'expriment autour de l'objet central. Le lecteur appréciera la construction de l'ouvrage, structuré par une introduction dense qui rappelle ce que son auteure nomme le " référent commun des auteurs " constitué par les savoirs accumulés au cours des trente dernières années sur les relations entre formation et emploi. De la même manière, les deux parties font l'objet d'une présentation utile permettant à cet ouvrage d'être bien plus qu'une collection de chapitres.

Pour exposer les thèses contenues, nous faisons le choix de distinguer trois registres. Les textes de Guy Brucy, Fabienne Maillard, Catherine Agulhon et Nathalie Moncel analysent des constructions socio-historiques de la relation formation-emploi. Tandis que ceux d'Emmanuel de Lescure, Nicolas Divert, Samuel Bouron et Ivan Chupin, Serge Katz et Gilles Moreau centrent leur approche sur l'étude d'un groupe professionnel et sa relation avec le système de formation. Enfin, les chapitres de José Rose, Bernard Fourcade et Bernard Prot sont résolument tournés vers l'action publique, " en l'adossant à une réflexion de recherche» (p. 77). 


\section{La relation formation-emploi au prisme des diplômes professionnels}

Les constructions socio-historiques de la relation formation-emploi sont analysées au prisme des diplômes professionnels. L'affichage de la mention " professionnelle ", qui figure dans les diplômes étudiés (CAP - certificat d'aptitude professionnelle, baccalauréat professionnel, licences et masters professionnels), ne vise pas uniquement à mettre en avant des savoir-faire de métier, qui seraient transmis dans le cours de la formation. Il est aussi le produit de la circulation d'acteurs et d'idées entre monde de l'éducation, milieux patronaux et sphère politique, dans un contexte de montée de l'incertitude sur les marchés du travail. La professionnalisation des diplômes est mise en avant dans les différents moments historiques relatés. Les auteurs mettent en évidence qu'au-delà des discours lénifiants, l'injonction à la professionnalisation agit comme un outil au service d'un mouvement plus général de réforme.

\section{Articuler formation et emploi selon les groupes professionnels}

L'entrée par les groupes professionnels révèle sa fécondité pour penser les articulations entre formation et emploi. Qu'on porte le regard sur les agents de la formation, sur les travailleurs de la mode, sur les journalistes, les comédiens ou encore les mécaniciens, force est de constater que les auteurs relativisent la place occupée par la formation et par la certification dans les régulations professionnelles. Si la certification ne conduit pas nécessairement à l'emploi pour lequel l'individu s'est formé, ceux qui occupent cet emploi ne sont pas toujours détenteurs de la certification. Agents de la formation, État, employeurs, représentants des salariés œuvrent collectivement et non sans antagonisme à un mouvement permanent de régulation-dérégulation, au gré des mutations socio-économiques générales ou sectorielles. C'est ce mouvement qui est analysé par les auteurs de cette partie.

\section{La relation formation emploi en termes de décision publique}

Trois auteurs mobilisent les apports de la recherche dans leur discipline pour étayer la décision publique. La clinique de l'activité est présentée comme un moyen de répondre aux dilemmes rencontrés dans la conception des référentiels d'activités professionnelles. Comment rendre compte de ce qu'est l'activité et quels sont les attendus en termes de formation? Ces interrogations classiques de la discipline sont de nature à nourrir la réflexion des concepteurs des référentiels. Par ailleurs, si José Rose propose d'améliorer les enquêtes d'insertion pour aider à la décision publique, Bernard Fourcade considère que cet exercice est aporétique tant l'insertion est un processus pluridimensionnel.

Depuis plus d'une trentaine d'année, l'accès à l'emploi se réalise dans une société de pleinchômage (Maruani, 2001). On assiste dans le même temps à une démocratisation de l'enseignement qui demeure ségrégative (Merle, 2002). Considérée comme une recette miracle par bon nombre de "réformateurs ", appelée de leurs vœux par les représentants des entreprises toujours prompts à pointer le décalage entre les besoins des entreprises et la formation des sortants du système éducatif, mobilisée à des fins stratégiques par diffé- 
rents responsables de programmes pédagogiques, attendue par les élèves et leurs parents inquiets de leur devenir professionnel, l'injonction à la professionnalisation des diplômes occupe une place de choix dans les questions sociales. Le premier mérite de cet ouvrage est de suivre différentes interrogations sur le sens des vocables mobilisés, sur l'effet de ces vocables, sur les mises en pratiques de cette injonction. Incontestablement, la réunion dans un même ouvrage des travaux sociologiques fondés sur des matériaux aussi bien quantitatifs que qualitatifs sur des terrains et objets variés est le deuxième point fort de cette entreprise collective. Enfin, le dialogue contradictoire permet la mise à distance des idées simplistes qui envahissent parfois le débat social. Un regret toutefois, à une exception près - le texte de Gilles Moreau - l'attention ne porte pas sur les bénéficiaires des formations et les titulaires des diplômes. Or, en tant qu' "agents agissant ", ces derniers contribuent aussi à façonner le triptyque formation-certification-insertion. La conclusion ouvre de nouvelles perspectives en appelant à porter le regard sur le droit et la juridicisation des rapports de travail et d'emploi ou par le développement de recherches comparatives à l'échelle internationale. On ne peut que souhaiter que ces travaux soient également attentifs aux différents échelons et à leur articulation : les niveaux de la régulation (cadre européen, État, région et territoire), les acteurs de la régulation (les pouvoirs publics, les entreprises, les représentants des salariés, agents de la formation, bénéficiaires), et les objets de cette régulation.

\section{Référence de l'ouvrage}

Maillard F. (dir.) (2012), Former, certifier, insérer. Effets et paradoxes de linjonction à la professionnalisation des diplômes, Presses Universitaires de Rennes, Rennes.

\section{Bibliographie}

Maruani M. (2001), «L'emploi dans une société de plein-chômage » in Pouchet A. (dir.) Sociologies du travail, quarante ans après, Ed. Elsevier, Paris, pp. 191-200.

Merle P. (2002), La démocratisation de l'enseignement, Paris, « Repères », La Découverte.

Tanguy L. (dir.) (1986), L'introuvable relation formation-emploi : Un état des recherches en France, La Documentation française, Paris. 
